\title{
Proteins other than the locus of enterocyte effacement-encoded proteins contribute to Escherichia coli 0157:H7 adherence to bovine rectoanal junction stratified squamous epithelial cells
}

Indira T Kudva ${ }^{1 *}$, Robert W Griffin ${ }^{2}$, Bryan Krastins ${ }^{3,6}$, David A Sarracino ${ }^{3,6}$, Stephen B Calderwood ${ }^{2,4,5}$ and Manohar John ${ }^{2,4,7}$

\begin{abstract}
Background: In this study, we present evidence that proteins encoded by the Locus of Enterocyte Effacement (LEE), considered critical for Escherichia coli O157 (O157) adherence to follicle-associated epithelial (FAE) cells at the bovine recto-anal junction (RAJ), do not appear to contribute to 0157 adherence to squamous epithelial (RSE) cells also constituting this primary site of 0157 colonization in cattle.

Results: Antisera targeting intimin- $\gamma$, the primary 0157 adhesin, and other essential LEE proteins failed to block O157 adherence to RSE cells, when this pathogen was grown in DMEM, a culture medium that enhances expression of LEE proteins. In addition, RSE adherence of a DMEM-grown-O157 mutant lacking the intimin protein was comparable to that seen with its wild-type parent $\mathrm{O} 157$ strain grown in the same media. These adherence patterns were in complete contrast to that observed with HEp-2 cells (the adherence to which is mediated by intimin- $\gamma$ ), assayed under same conditions. This suggested that proteins other than intimin- $\gamma$ that contribute to adherence to RSE cells are expressed by this pathogen during growth in DMEM. To identify such proteins, we defined the proteome of DMEM-grown-0157 (DMEM-proteome). GeLC-MS/MS revealed that the O157

DMEM-proteome comprised 684 proteins including several components of the cattle and human 0157 immunome, orthologs of adhesins, hypothetical secreted and outer membrane proteins, in addition to the known virulence and LEE proteins. Bioinformatics-based analysis of the components of the 0157 DMEM proteome revealed several new O157-specific proteins with adhesin potential.

Conclusion: Proteins other than LEE and intimin- $y$ proteins are involved in 0157 adherence to RSE cells at the bovine RAJ. Such proteins, with adhesin potential, are expressed by this human pathogen during growth in DMEM. Ongoing experiments to evaluate their role in RSE adherence should provide both valuable insights into the O157-RSE interactions and new targets for more efficacious anti-adhesion 0157 vaccines.
\end{abstract}

Keywords: O157, Rectoanal junction, LEE, Adherence, DMEM, GeLC-MS/MS

\footnotetext{
* Correspondence: Indira.Kudva@ars.usda.gov

${ }^{1}$ Food Safety and Enteric Pathogens Research Unit, National Animal Disease Center, Agricultural Research Service, U.S. Department of Agriculture, Ames, lowa 50010, USA

Full list of author information is available at the end of the article
} 


\section{Background}

Escherichia coli (E. coli) O157 (O157) was first identified as a human enteric pathogen in 1982 and has since been implicated in several outbreaks and sporadic infections $[1,2]$. Currently, this human pathogen ranks fourth after Campylobacter, Salmonella, and Shigella among the etiologic agents causing diarrhea in North America [3,4]. Cattle are the primary reservoirs for O157, with the bovine recto-anal junction (RAJ) serving as the primary colonization site for O157. Humans acquire infection by consumption of undercooked beef products such as ground meat or foods contaminated with manure $[1,2]$.

The bovine RAJ comprises of two cell types, the follicle associated epithelium (FAE) towards the distal colon and the stratified squamous epithelium (RSE) closer to the anal canal [5]. Thus far, studies analyzing O157 persistence at the RAJ have focused primarily on its interactions with the FAE cells [6,7]. Proteins encoded on the O157 pathogenicity island, Locus of Enterocyte Effacement (LEE), have been shown to play a critical role in O157 adherence to FAE cells. These include the E. coli secreted proteins EspA and EspB, the adhesin Intimin, and the translocated receptor for Intimin, Tir which is secreted via the LEE-encoded type III secretion system (TTSS) [6-8]. Hence, several pre-harvest control measures being evaluated in cattle to control or eliminate O157 from entering the food chain [9-14], include vaccines targeting these LEE-encoded proteins. For instance, Potter et al. developed a vaccine comprising wild-type O157 culture supernatants that contain the TTSS proteins, Tir and Esps [15]; however, similar protection was noted in animals inoculated with the culture supernatant from a mutant strain of $\mathrm{O} 157$ lacking the tir gene. In addition, the immune response of the vaccinated animals was not merely to the TTSS proteins but also against a number of other proteins that were present in the supernatant. Interestingly, although the vaccine decreased both the number of E. coli $\mathrm{O} 157$ shed in the feces of vaccinated animals, and those colonizing the terminal rectum, it did not reduce the duration of shedding despite the subcutaneous administration of three doses of the vaccine $[15,16]$; www.bioniche.com. Similar results were also observed with another vaccine that targets the O157 siderophore receptor and porin (SRP) proteins $[17,18]$; https://animalhealth.pfizer.com. This clearly suggests that unidentified proteins other than those constituting the TTSS or SRP may play a crucial role in bovine colonization, and that the identification and inclusion of such proteins is likely to increase the efficacy of vaccines for elimination of $\mathrm{O} 157$ from the gastrointestinal tracts of cattle. Further supporting this inference are reported observations that the TTSS proteins do not significantly contribute to the persistence of O157 in feedlot cattle [19], or to O157 invasion of crypt cells at the RAJ [20].
In a previous study, O157 was observed to adhere to RSE cells in vivo and in vitro, besides the FAE cells [5] and this observation was used to develop a unique in vitro adherence assay for $\mathrm{O} 157$ with RSE cells [5]. In this study, we decided to (i) evaluate if the LEE-encoded proteins would also be critical for O157 adherence to RSE cells, as for FAE cells, and (ii) in the event that these proteins would not play a significant role in RSE cell adherence, define the proteome of $\mathrm{O} 157$ as expressed when grown in the adherence assay media, DMEM, to assemble targets for future evaluation in RSE adherence. Experimental and bioinformatic evaluation of such targets could in fact help identify a subset of novel adhesins that may have excellent potential to increase the efficacy of the anti-adhesion, cattle O157 vaccines, by eliminating O157 from both FAE and RSE cells at the RAJ.

\section{Methods}

\section{Bacterial strains and culture conditions}

The wild-type O157 strain EDL933 (O157), a sequenced isolate implicated in human disease [21], was used in this study. We cultured O157 in Dulbecco Modified Eagle Medium-Low Glucose (DMEM; Gibco/lnvitrogen Corporation, Grand Island, NY), for the cell adherence assays described below. The rationale for the use of this culture medium was (i) to reflect the growth conditions used in the eukaryotic cell adherence assays; and (ii) to closely parallel the in vivo nutrient-limiting conditions, and conditions used to prepare the cattle-use approved, LEE protein based, anti-adhesion O157 vaccine. In addition, another wild-type $\mathrm{O} 157$ strain 86-24 (86-24), its isogenic mutant (86-24eae $\Delta 10)$ negative for Intimin, and this mutant complemented with the plasmid pEB310 (86-24eae $\Delta 10(\mathrm{pEB} 310))$ expressing Intimin, were also tested in the adherence assay [22]. The 86-24 strain and its derivatives were obtained from Dr. A. D. O’Brien, Uniformed Services University of the Health Sciences, Bethesda, MD.

We also cultured O157 in DMEM for proteomic analysis. Specifically, an overnight culture of the wild-type O157 strain in Luria-Bertani (LB) broth was pelleted and washed with sterile phosphate buffered saline (PBS; $\mathrm{pH}$ 7.4), and subcultured to an initial $\mathrm{OD}_{600}$ of 0.05 in fresh DMEM. After incubation at $37{ }^{\circ} \mathrm{C}$ with shaking at $250 \mathrm{rpm}$ to an $\mathrm{OD}_{600}$ of 0.8 to 1.0 , cells were harvested by centrifugation at 7,000 rpm, $15 \mathrm{~min}$ at $4{ }^{\circ} \mathrm{C}$. Cells were washed three times with an equal volume of sterile PBS ( $\mathrm{pH} 7.4)$, and processed to obtain cell lysate and pellet fractions for proteomic analysis as previously described [23].

0157-RSE cell adherence inhibition assay: (i) in the presence of pooled anti-LEE proteins, anti-intimin and anti-H7 antisera

Adherence of O157 to the RSE cells was previously demonstrated and developed into an adherence assay in 
our laboratory [5]. In this study, the ability of pooled, rabbit polyclonal antisera to interfere with and inhibit O157 adherence to RSE cells was evaluated. Specifically, antisera generated against the recombinant LEE-encoded proteins, Tir, EspA and EspB, and Intimin, in rabbits (National Animal Disease Center Stocks), was pooled. Rabbit antisera targeting the $\mathrm{O} 157$ flagellar antigen $\mathrm{H7}$ (Difco Laboratories, Inc., Detroit, MI) was also mixed into the pooled antisera, which was then tested at 1:5 and 1:10 dilutions. Specificity was confirmed by reacting each antiserum against both $\mathrm{O} 157$ cell lysates and the cognate protein in western blotting experiments (data not shown). Rabbit sera (Sigma-Aldrich, St. Louis, MO) from healthy animals (normal rabbit sera), at a 1:5 dilution, was used as a control.

\section{(ii) In the presence of anti-Intimin antisera alone}

To specifically evaluate the role of intimin, the rabbit anti-Intimin antisera was evaluated separately for its ability to prevent O157 adherence to RSE cells at 1:5 and 1:10 dilutions.

Each of the RSE adherence assays was conducted in 8 technical and 2 biological replicates as described previously [5], with minor modifications, as follows. RSE cells were washed and resuspended in $1 \mathrm{ml}$ Dulbecco Modified Eagle Medium - No Glucose (DMEM-NG) $\pm 2.5 \%$ D + Mannose, in $16 \times 100 \mathrm{~mm}$ glass tubes, to a final concentration of $10^{5}$ cells $/ \mathrm{ml}$. Although Type 1 fimbriae are not expressed by $\mathrm{O} 157$, we included $\mathrm{D}+$ Mannose in parallel assays to cover any hitherto unknown transient expression especially in mutant strains. Bacterial pellets from overnight cultures in DMEM, incubated at $37^{\circ} \mathrm{C}$ without aeration, were resuspended in sterile saline with or without antisera ('no sera' control), and incubated at $37^{\circ} \mathrm{C}$ for $30 \mathrm{~min}$. The bacteriaantibody mix was then added to the RSE cells suspension to final bacteria:cell ratio of 10:1, and the mixture incubated with aeration $\left(37^{\circ} \mathrm{C}, 110 \mathrm{rpm}\right.$, for $\left.4 \mathrm{~h}\right)$. At the end of $4 \mathrm{~h}$, the mixture was pelleted and washed thoroughly, once with $14 \mathrm{ml}$ DMEM-NG, and twice with $14 \mathrm{mls}$ of sterile, distilled water $\left(\mathrm{dH}_{2} \mathrm{O}\right)$ before reconstituting in $100 \mu \mathrm{ld} \mathrm{H}_{2} \mathrm{O}$. Eight $2 \mu \mathrm{l}$ drops of this suspension were placed on Polysine (Thermo Scientific Pierce) slides and dried overnight under direct light to quench non-specific fluorescence, before fixing in cold 95\% ethanol for $10 \mathrm{~min}$. The slides were then stained with $1 \%$ toluidine blue, or with fluorescence-tagged antibodies that specifically target O157 and the RSE cell cytokeratins as described previously [5]. Each experiment was then done in duplicate.

O157 adherence patterns on RSE cells were recorded as diffuse, or aggregative (clumps) for all positive interactions that involved direct association with the cells [5]. Scattered bacteria and bacterial micro-colonies not adhering to cell membranes were considered to be negative for adherence to the epithelial cells [5]. A total of 100-
160 well dispersed RSE cells (10-20 cells per drop or chamber) were analyzed per slide as described previously [5]. The percent RSE cells with and without bacteria adhering to them were determined [5]. If more than 50\% of RSE cells had $>10$ bacteria attached, the adherence was recorded as strongly positive. For $>50 \%$ RSE cells with 1-10 adherent bacteria, the adherence was recorded as moderately positive. For less than 50\% RSE cells with 1-5 adherent bacteria, the result was recorded as non-adherent.

\section{7-HEp-2 cell adherence inhibition assay}

The role played by LEE-encoded proteins and Intimin in the adherence of $\mathrm{O} 157$ to HEp-2 cells, has already been defined previously [22] and hence, this assay was used for comparative reasons. The assay was conducted as described previously [5] except that, the washed bacterial pellets were incubated with or without antisera ('no sera' control), at $37^{\circ} \mathrm{C}$ for $30 \mathrm{~min}$, prior to addition to the HEp-2 cells. Both the pooled antisera and anti-intimin antisera, as described above, were used at dilutions ranging from 1:5 to 1:100 in these assays. Each assay was conducted in duplicate, and in 3-6 chambers of the chamber slides per run. Slides were stained with $1 \%$ toluidine blue, or with fluorescence-tagged antibodies that specifically target $\mathrm{O} 157$ and the HEp-2 cell actin filaments as described previously [5] and adherence patterns recorded as for RSE cells (see above).

\section{Adherence of 86-24, 86-24eae $\Delta 10$, and 86-24eae $\Delta 10$ (pEB310), to RSE and HEp-2 cells}

Wild-type 86-24 and its mutant derivatives were used to verify the role of Intimin directly and compare the results with that of the O157 adherence inhibition assays. This assay was conducted, recorded, as previously described and done in the absence of any antisera [5].

\section{OneDimensional (1D) SDS-PAGE liquid chromatography tandem mass spectrometry (GeLC-MS/MS)}

Top down proteomic analysis was done at the Harvard Partners Center for Genetics and Genomics, Cambridge, Massachusetts. O157 cell pellet and lysate fractions were concentrated using spin filters (MW cutoff 5000 Daltons; Vivascience Inc., Englewood, NY), fractionated on 1D SDS-PAGE, and digested in-gel with trypsin prior to tandem mass spectrometry (MS/MS) as described previously [23]. The rationale for incorporating a 1D SDSPAGE fractionation step is that this modification reduces complexity of protein mixtures, permits a larger dynamic range of protein identification, and allows for significantly better reproducibility [24,25].

For mass spectrometry (MS), samples were subjected to three different runs on an LCQ DECA XP plus Proteome $\mathrm{X}$ workstation (LCQ) from Thermo Finnigan as 
described earlier [23,26]. For each run, $10 \mu \mathrm{L}$ of each reconstituted sample was injected with a Famos Autosampler, and the separation was done on a $75 \mu \mathrm{m}$ (inner diameter) x $20 \mathrm{~cm}$ column packed with $\mathrm{C}_{18}$ media running at a flow rate of $0.25 \mu \mathrm{l} / \mathrm{min}$ provided from a Surveyor MS pump with a flow splitter with a gradient of water, $0.1 \%$ formic acid and then $5 \%$ acetonitrile, $0.1 \%$ formic acid (5\%-72\%) over the course of $480 \mathrm{~min}(8.0$ hour run). Between each set of samples, a standard of a 5 Angiotensin mix of peptides (Michrom BioResources) was run to ascertain column performance, and to observe any potential carryover that might have occurred. The LCQ was run in a top five configuration, with one MS scan and five MS/MS scans. Dynamic exclusion was set to 1 with a limit of 30 seconds.

Peptide identifications were made using SEQUEST (Thermo Finnigan) through the Bioworks Browser 3.2, as described previously [23]. Sequential database searches were performed using the O157 strains EDL933 and Sakai FASTA database from European Bioinformatics Institute http://www.ebi.ac.uk/newt/display using static carbamidomethyl-modified cysteines and differential oxidized methionines. These protein databases (Escherichia coli (strain Sakai/O157:H7/RIMD 0509952/ EHEC) - Tax ID: 386585 and Escherichia coli (strain EDL933/ATCC 700927/O157:H7/EHEC) - Tax ID: 155864) have a total of 10,737entries. A reverse O157 strain EDL933 FASTA database was spiked in to provide noise and determine validity of the peptide hits, so that known and theoretical protein hits can be determined without compromising the statistical relevance of all the data [26]. The MS data was searched with a 2-Dalton window on the MS precursor with a 0.8 Dalton on the fragment ions. Peptide score cutoff values were chosen at crosscorrelation values (Xcorr) of 1.8 for singly charged ions, 2.5 for doubly charged ions, and 3.0 for triply charged ions, along with delta rank scoring preliminary cutoff (deltaCN) values of 0.1 , and cross-correlation normalized values (RSp) of 1 . The cross-correlation values chosen for each peptide assured a high confidence match for the different charge states, while the deltaCN values ensured the uniqueness of the peptide hit. The RSp value of 1 ensured that the peptide matched the top hit in the preliminary scoring. At these peptide filter values, very few reverse database hits were observed, which permitted a higher confidence in the few single peptide protein identifications. Furthermore, single hit proteins were manually validated to ensure relevance.

\section{Bioinformatics}

Cellular location of proteins was determined using amino acid sequences of cognate proteins in the $\mathrm{O} 157$ sequence databases at http://www.ncbi.nlm.nih.gov/protein. In addition, extracytoplasmic proteins were verified for the presence of signal sequences using the program SignalP 3.0 at http://www.cbs.dtu.dk/services/SignalP, and subcellular localization of other proteins confirmed using the PSORT/PSORT-B program (http://psort.nibb. ac.jp/). Putative functions were determined by querying the Conserved Domain Database (CDD) at http://www. ncbi.nlm.nih.gov/Structure/cdd/wrpsb.cgi Protein components of the O157 DMEM-proteome with adhesion potential were shortlisted using Vaxign, a reverse vaccinology based vaccine target prediction and analysis system at http://www.violinet.org that utilizes the SPAAN algorithm [27].

\section{Results and discussion}

LEE- encoded proteins considered critical for O157 adherence to FAE cells at the RAJ did not appear to have a role in O157 adherence to RSE cells at this same site. Both O157 strains grown in DMEM and pre-incubated with pooled, polyclonal antisera generated against the LEE (Tir, EspA, EspB, and Intimin) and flagellar $\mathrm{H} 7$ proteins, or the anti-Intimin antisera alone, at 1:5 and 1:10 dilution, continued to adhere to the RSE cells, irrespective of the presence/absence of $\mathrm{D}+$ Mannose. Data is shown for one of the $\mathrm{O} 157$ strains in the presence of D+ Mannose (Additional file 1, Figure 1, panel A, Figure 2). These results were consistent between all trials, irrespective of toluidine blue or immunofluorescent staining, and did not show any differences in the adherence patterns compared to the controls. The same O157RSE cell-adherence pattern was observed in the controls with normal rabbit sera added at 1:5 dilution (data not shown), and in the absence of any sera (Additional file 1, Figure 1, panel B; Figure 2) [5], irrespective of the presence/absence of D + Mannose. The continued adherence of O157 to the RSE cells in the presence of antibodies to the LEE proteins may have been due to the masking of these antigens and the unmasking of other O157 adhesins targeting the receptors on the RSE cells. To that effect an increase in the total number of RSE cells with adherent bacteria and decrease in the total number of RSE cells with no adherent bacteria was observed, in the presence of pooled and anti-Intimin antisera (Figure 2). We intentionally included antisera targeting the flagellar antigen $\mathrm{H} 7$ as flagella have been demonstrated to play a role in initial adherence to plant cells and the FAE $[28,29]$. These results suggest that additional mechanisms of adherence, distinct from those attributable to LEE, Intimin and flagellar $\mathrm{H} 7$ proteins, are involved in $\mathrm{O} 157$ attachment to the RAJ squamous epithelial cells.

On the other hand, the LEE-encoded proteins were critical to O157 adherence to HEp-2 cells as demonstrated previously [22], with or without D + Mannose. As shown in Additional file 2 and Figure 3, panel A and Figure 2, 


\section{A}

Pooled antisera at 1:5 dilution
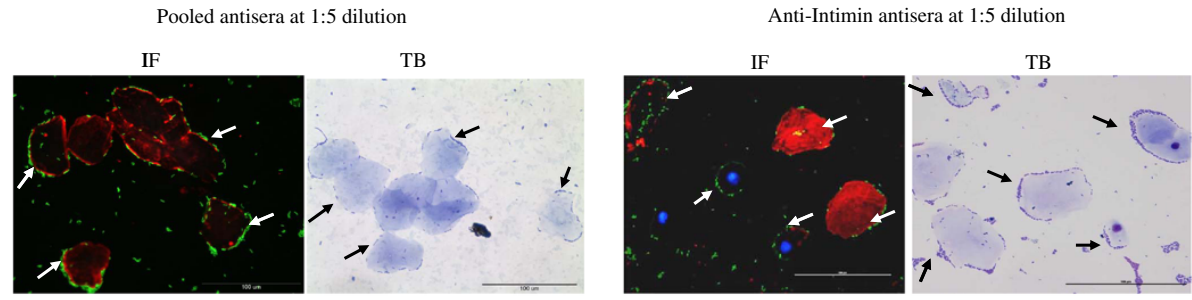

B

No antisera

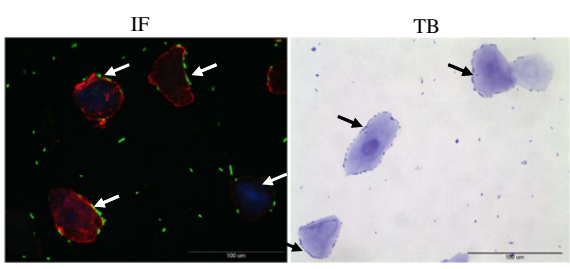

Figure 1 Adherence patterns of 0157 strain EDL 933 on RSE cells, in the presence of D+ Mannose and +/- antisera. Panel A, in the presence of "pooled antisera" against LEE, Intimin and flagellar H7 proteins, and the anti-Intimin antisera alone, at 1:5 dilutions. Panel B, in the absence of any sera (No sera). The immunofluorescence (IF) stained slides are shown at 40x magnification. 0157 have green fluorescence, cytokeratins' of RSE cells have orange-red fluorescence, and their nuclei have blue fluorescence. The arrows in the adjacent toluidine blue (TB) stained slides, at 40x magnification, point to RSE-adherent O157.

preincubation with the pooled polyclonal antisera and the anti-Intimin antisera significantly interfered with and prevented $\mathrm{O} 157$ adherence to HEp-2 cells, at all dilutions tested. An increase in number of HEp-2 cells without any adhering bacteria was observed in the presence of either antiserum, accordingly (Figure 2). However, pre-incubation with normal rabbit sera at 1:5 dilution (data not shown) showed the same diffuse,

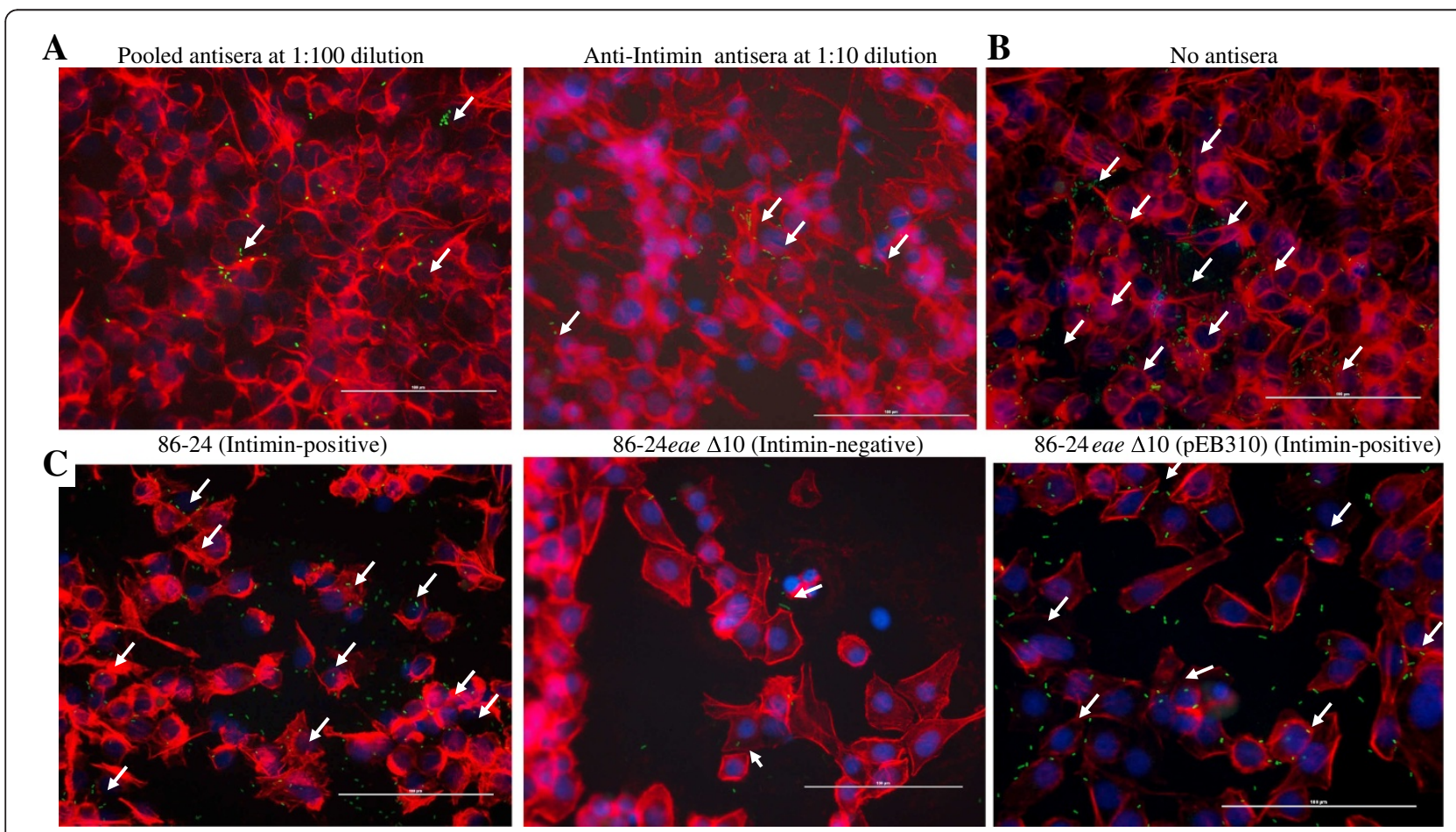

Figure 2 Quantitative representation of the adherence patterns of 0157 strains EDL 933, and 86-24 along with its mutant derivatives, on RSE and HEp-2 cells. Percent mean \pm standard error of mean of cells with adherent bacteria or no bacteria, in the ranges shown in the legend, are depicted in each graph. 


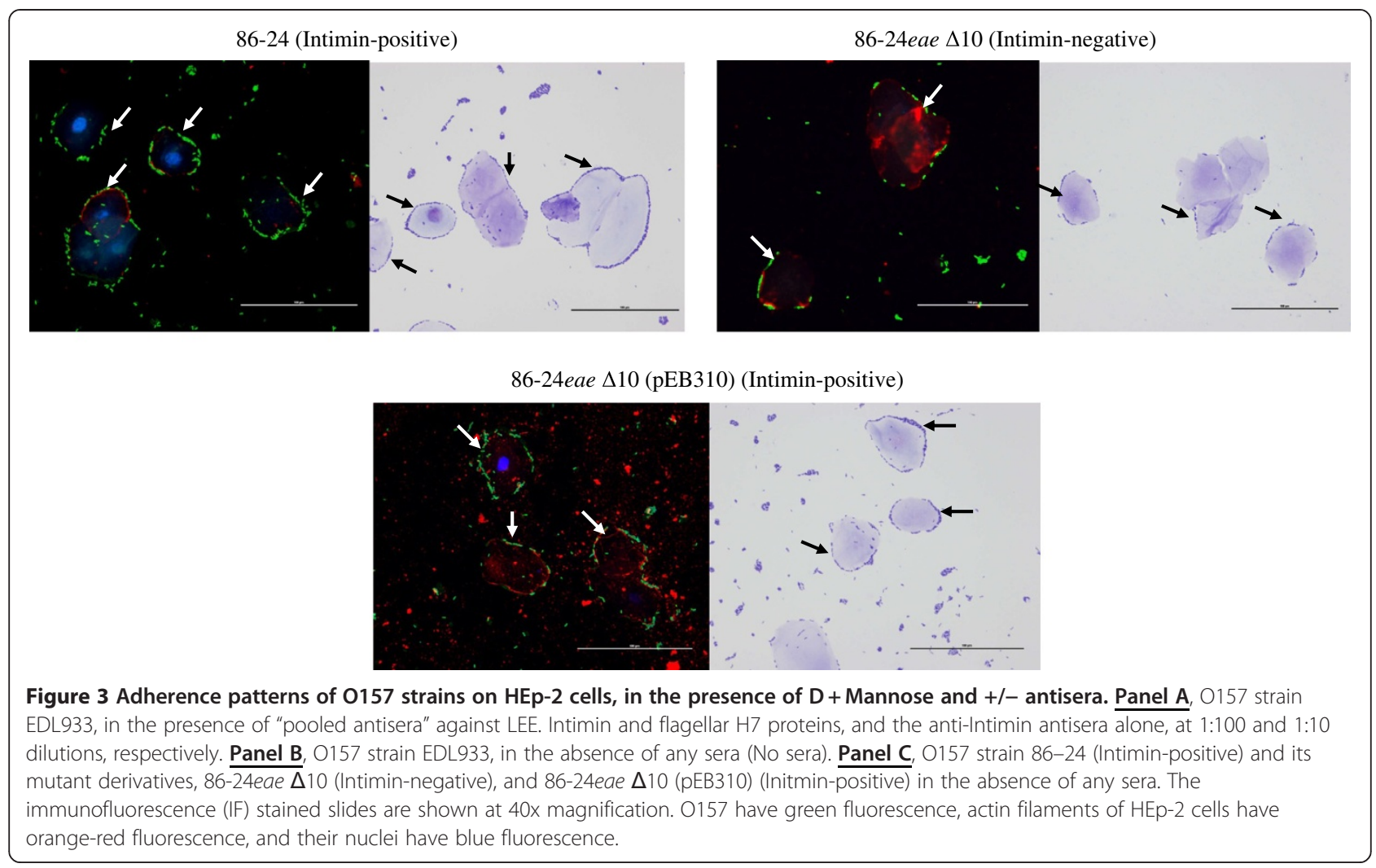

moderate adherence as in the absence of any antisera (Additional file 2, Figure 3 panel B and Figure 2).

The results observed with the adherence inhibition assays were further verified by the adherence patterns of O157 strain 86-24 (86-24) and its mutant derivatives on HEp-2 and RSE cells (Figure 3, panel C, Figures 4 and 2). The intimin-negative mutant 86-24eae $\Delta 10$ did not adhere well to the HEp-2 cells compared to the intimin-positive, wild-type 86-24 or complemented mutant, 86-24eae $\Delta 10$ (pEB310) that demonstrated diffuse, moderate adherence (Figure 3, panel C, Figure 2, and Additional file 2). Actin accumulation observed in the majority of HEp-2 cells with 100x magnification only in the presence of 86-24 and 86-24eae $\Delta 10$ (pEB310), along with an increase in the number of HEp-2 cells without adhering bacteria in the presence of 86-24eae $\Delta 10$, further verified these observations (data not shown). This confirmed the role of intimin in $\mathrm{O} 157$ adherence to HEp-2 cells. On the otherhand, 86-24 and all its mutant derivatives demonstrated diffuse, strong adherence to RSE cells, irrespective of intimin expression (Figures 4 and 2 , and Additional file 1). Infact with 86-24eae $\Delta 10$, the number of RSE cells with adhering bacteria actually increased, which suggested that intimin did not have a role in the adherence of O157 to RSE cells.

In order to verify if proteins other than LEE proteins were being expressed by $\mathrm{O} 157$ upon growth in DMEM which could have a possible role in $\mathrm{O} 157$ adherence to RSE cells, we analyzed the $\mathrm{O} 157$ proteome as expressed in DMEM. While the proteome of $\mathrm{O} 157$ has been analyzed under various other growth conditions [30-33] we decided to evaluate the same following growth in DMEM for several reasons, such as (i) this was the media used to culture both bacteria and the RSE cells, separately, prior to the adherence assays, (ii) the media closely mimicked the nutrient-limiting conditions seen in vivo, and (iii) this media closely matched that used to develop a commercially available cattle, O157 vaccine $[15,16$; www.bioniche. com]. Our observations did not support a role for other host (RSE-cell)-derived factors in this adherence of $\mathrm{O} 157$ and hence, we did not evaluate RSE-cell adherence of O157 cultured in eukaryotic cell-conditioned media. This inference came from the fact that similar adherence results were obtained when DMEM was supplemented with norepinephrine (NE; DMEM-NE), a host neuroendocrine hormone that is encountered by $\mathrm{O} 157$ in vivo during the actual process of infection (data not shown). NE is reportedly a mimic of autoinduer 3 (AI-3), which regulates O157 virulence gene expression via quorum sensing [34]. Further, Intimin, its receptor, Tir, as well as EspB were expressed in equivalent amounts in both DMEM and DMEM-NE, as observed using western blotting by others [34], and by us, and also using top down proteomics by us (data not shown). 


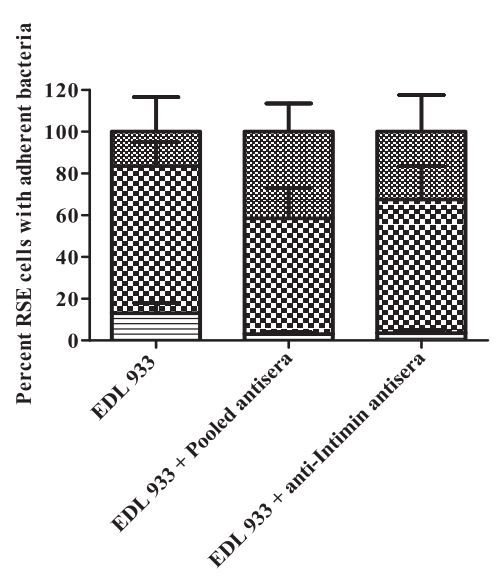

Bacteria interacting with RSE cells

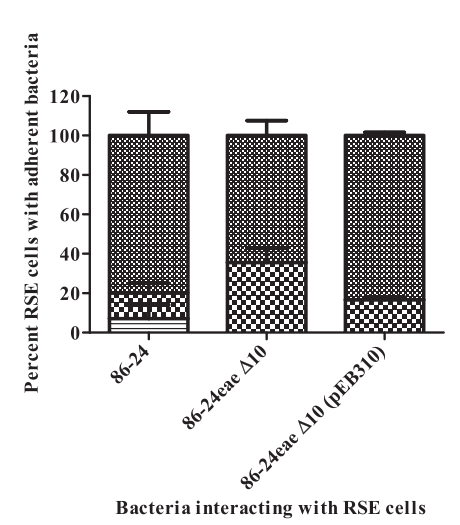

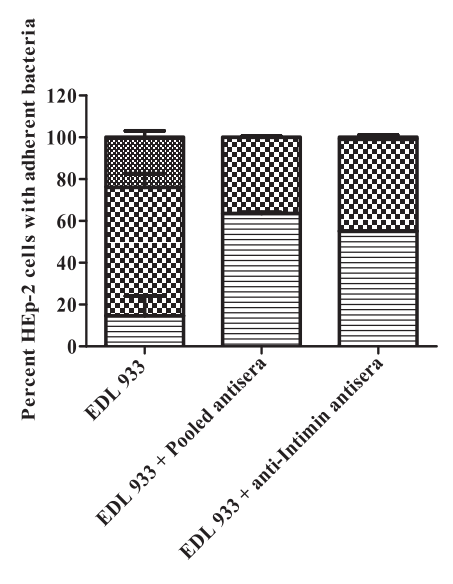

Bacteria interacting with HEp-2 cells

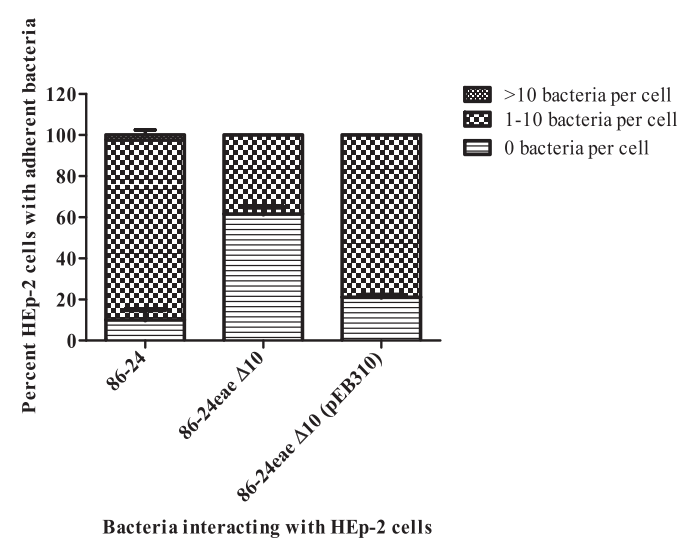

Figure 4 Adherence patterns of 0157 strain 86-24 (Intimin-positive) and its mutant derivatives, 86-24eae $\Delta 10$ (Intimin-negative) and 86-24eae $\Delta 10$ (pEB310) (Initmin-positive), on RSE cells, in the presence of D + Mannose. The immunofluorescence (IF) stained slides are shown at 40x magnification. O157 have green fluorescence, cytokeratins' of RSE cells have orange-red fluorescence, and their nuclei have blue fluorescence. The arrows in the adjacent toluidine blue (TB) stained slides, at 40x magnification, point to RSE-adherent O157.

A total of 684 proteins were identified as being part of the O157 DMEM-proteome (13\% of the O157 sequenced proteome), and these included several characterized and hypothetical/unknown proteins besides the TTSS proteins. While 171 of these proteins were uncharacterized with hypothetical functions assigned in the $\mathrm{O} 157$ genome [21;
Figure 5, Additional files 3 and 5-12], the remaining 513 proteins localized to various bacterial cell compartments with functions including metabolic, cell division, regulatory, transport, environmental adaptation, and previously characterized $\mathrm{O} 157$ virulence factors [21]; Figure 5, Additional files 4 and $5,6,7,8,9,10,11,12$. Proteins

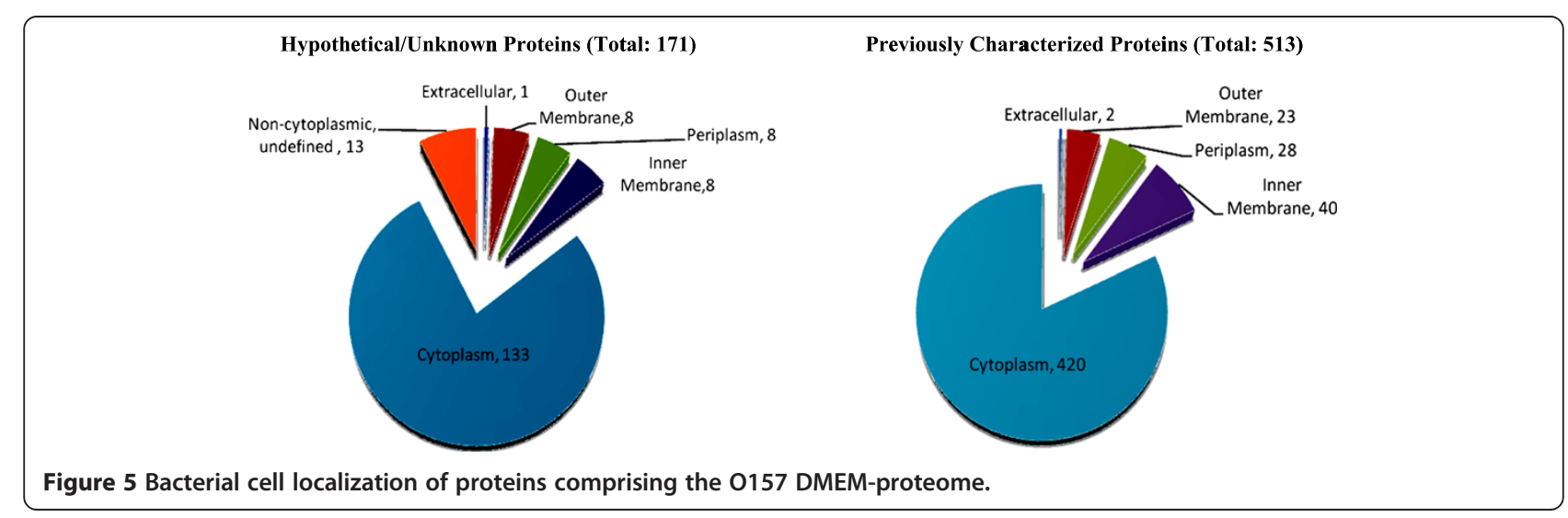


Table 1 Bioinformatically determined putative adhesins in the 0157 DMEM-Proteome Protein Identity: Sequences not homologous [O-island] OR Peptide Bacterial Cell homologous [Backbone] to E. coli K12 MG1655

$\begin{array}{ll}\text { Peptide } & \text { Bacterial Cell } \\ \text { Hits } & \text { Localization }\end{array}$

Proteins Proteins Proteins
identified identified associated by PELS ${ }^{1}$ by IVIAT viulence

Tir: Translocated intimin receptor protein: $\mathbf{0}$-island\#148

Eae: Intimin: Adhesin; attaching and effacing protein: O-island \#148

ChuA: Outer membrane heme/hemoglobin receptor; heme utilization/transport protein: O-island \#140

EspP: Serine protease; secreted autotransporter: p0157

TerE: Putative tellurium resistance protein B (Putative phage inhibition, colicin resistance): $\mathbf{0}$-island \#43

Z2239: Putative outer membrane porin protein: O-island \#62

FimG: Fimbrial Morphology: Backbone

MsrA: Peptide methionine sulfoxide reductase : Backbone

Yjel: Putative lipoprotein :Backbone

BtuB: Outer membrane receptor for transport of vitamin B12,

E colicins, and bacteriophage: Backbone

DsbA: Thiol:disulfide interchange protein precursor : Backbone

PstS: High-affinity phosphate-specific transport system;

periplasmic phosphate-binding protein: Backbone

YiaF: Hypothetical protein: Backbone

Slp: Outer membrane protein induced after carbon starvation: Backbone

LivJ: Leu/lle/Nal-binding protein precursor; $A B C$ transporter: Backbone

PpiA: Peptidyl-prolyl cis-trans isomerase A precursor : Backbone

YrbD: Hypothetical protein precursor; probable phospholipid

$\mathrm{ABC}$ transporter-binding protein MlaD :Backbone

YraP: Hypothetical protein; putative transport :Backbone

TolC: Outer membrane channel; specific tolerance to colicin

E1; segregation of daughter chromosome: Backbone

Lpp: Murein-lipoprotein; major outer membrane lipoprotein

precursor: Backbone

NlpB: Lipoprotein-34: Backbone

Z3508: Hypothetical protein :Backbone

OmpC: Outer membrane protein 1b: hyperosmotic shock: Backbone

YehZ: Putative transport system permease protein :Backbone

CspC: Cold shock-like protein : Backbone

CspD: Cold shock-like protein : Backbone

CspE: Cold shock-like protein : Backbone

YeaF: Hypothetical protein yeaF; putative scaffolding protein

in the formation of a murein :Backbone

OsmE: Osmotically inducible lipoprotein E precursor; activator of ntr-like gene : Backbone

SlyB: Outer membrane lipoprotein slyB precursor : Backbone

YciD: Putative outer membrane protein :Backbone

Ycel: Putative GTP binding :Backbone

Artl: Arginine 3rd transport system periplasmic binding protein: Backbone YbiS: Putative transpeptidase :Backbone

3

36

\section{Extracellular}

Outer Membrane

Outer Membrane

Outer Membrane

Cytoplasm

Outer Membrane

Inner Membrane

Cytoplasm

Non-cytoplasmic

Outer Membrane

\section{Periplasm}

Periplasm

$$
\text { Cytoplasm }
$$

Outer Membrane

$$
\text { Periplasm }
$$

Cytoplasm

Periplasm

\section{Periplasm}

Outer Membrane

Outer Membrane

MepA, MItB, Slt

Outer Membrane

Non-cytoplasmic

Outer Membrane

Cytoplasm

Cytoplasm

Cytoplasm

Cytoplasm

Outer Membrane

$+$

YeaA

Cytoplasm

Outer Membrane

Outer Membrane

Non-cytoplasmic

Periplasm

Non-cytoplasmic 
Table 1 Bioinformatically determined putative adhesins in the 0157 DMEM-Proteome (Continued)

\begin{tabular}{lll}
\hline OmpX: Outer membrane protein X precursor : Backbone & 22 & Outer Membrane \\
FepA: Receptor for ferric enterobactin (Enterochelin) and colicins & 29 & Outer Membrane \\
B and D: Backbone & & +
\end{tabular}

${ }^{1}$ PELS: Proteomics- based Expression Library Screening

${ }^{2}$ IVIAT:In Vivo-Induced Antigen Technology

associated with $\mathrm{O} 157$ virulence or adherence in the DMEM-proteome included Tir, Intimin, EspB, LuxS, Iha, OmpA, KatP, ChuA, EspP, Stx1A, Stx1B, and Stx2B [20]; Additional files 4 and 5, 6, 7, 8, 9, 10, 11, 12. Interestingly, 64 of the $684(9.4 \%)$ proteins comprising the O157 DMEM-proteome were also part of the O157 immunoproteome in cattle, defined using the innovative proteome mining tool, Proteomics- based Expression Library Screening (PELS) [23]; Additional files 3 and 4. In addition, nine members of the DMEM-proteome were also part of the O157 immunome in humans [26].

Given that a subset of pathogen proteins targeted by the host immune system help pathogens overcome hostile in vivo environments and rapidly adapt to host niches, counter host defenses, survive, propagate, and establish infection, it is very likely that proteins other than LEE proteins in the O157 DMEM-proteome play a role in O157 adherence to the RSE cells at the RAJ. The identification and inclusion of such proteins in anti-adhesion vaccine preparations, and their optimal administration to the host immune system, may enhance the efficacy of such vaccines in reducing or eliminating $\mathrm{O} 157$ not only from the FAE cells but also from the RSE cells of the bovine RAJ. Our strategy for selecting proteins constituting the O157 DMEM proteome with potential to function as adhesins for further evaluation was to employ the SPAAN algorithm [27], which is part of the web-based vaccine target prediction and analysis system at http://www.violinet.org. In particular, we shortlisted proteins that were shared by four sequenced O157 strains, namely, strain EDL933, strain EC4115, strain Sakai, and strain TW14359. Our analysis identified 36/684 components of the O157 DMEMproteome to have adhesin potential, including the extensively reported primary $\mathrm{O} 157$ adhesin, intimin- $\gamma$, and its protein receptor, Tir (Table 1). Three O157-specific proteins, namely ChuA, TerE, and a putative outermembrane porin protein on O-island \#62 (Table 1), have been prioritized and are under experimental evaluation for a role in O157 adherence to RSE cells. Also, towards studies of common STEC adherence mechanisms, we have determined homologs of several of these putative adhesins including those encoded on O-islands such as, Tir, Intimin, ChuA, TerE, EspP, bioinformatically (psiBLAST, National Center for Biotechnology Information; www.ncbi.nih.gov), in sequenced non-O157 Shiga-toxinproducing Escherichia coli (STECs) of clinical importance. Experimental evaluation of the contribution of these homologs to adherence of these non-O157 STEC, E. coli O26:H11, O103:H2 and O111:NM, are underway.

\section{Conclusion}

Proteins other than the LEE-encoded proteins are involved in O157 adherence to RSE cells at the bovine RAJ. Such proteins, with adhesin potential, are expressed by this human pathogen during growth in vitro, in DMEM. Three such putative adhesins, namely, ChuA, TerE, and a putative outermembrane porin protein on $\mathrm{O}$-island \#62, are currently under evaluation for their adherence potential. Such studies should provide both valuable insights into the O157-RSE interactions and new targets for more efficacious anti-adhesion O157 cattle vaccines.

\section{Additional files}

Additional file 1: http://www.biomedcentral.com/imedia/ 9899042126754199/supp1.pdf. TABLE A Quantitation of RSE cells with adherent bacteria in the presence of D + mannose.

Additional file 2: http://www.biomedcentral.com/imedia/ 6766700936754199/supp2.pdf. TABLE B Quantitation of HEp-2 cells with adherent bacteria in the presence of $D+$ mannose.

Additional file 3: http://www.biomedcentral.com/imedia/ 1105071156754199/supp3.pdf. TABLE C Uncharacterized hypothetical proteins of the 0157 DMEM-Proteome.

Additional file 4: http://www.biomedcentral.com/imedia/ 1751063870675419/supp4.pdf. TABLE D Previously characterized proteins of the 0157 DMEM-Proteome.

Additional file 5: http://www.biomedcentral.com/imedia/ 1777785157675419/supp5.pdf. DATA SHEETS: O157-DMEM MS/MS data sheet 1 .

Additional file 6: http://www.biomedcentral.com/imedia/ 1707955235675419/supp6.pdf. DATA SHEETS: O157-DMEM MS/MS data sheet 2 .

Additional file 7: http://www.biomedcentral.com/imedia/ 1451425738675419/supp7.pdf. DATA SHEETS: O157-DMEM MS/MS data sheet 3 .

Additional file 8: http://www.biomedcentral.com/imedia/ 3116488396754199/supp8.pdf. DATA SHEETS: O157-DMEM MS/MS data sheet 4 .

Additional file 9: http://www.biomedcentral.com/imedia/ 1233524502675419/supp9.pdf. DATA SHEETS: O157-DMEM MS/MS data sheet 5 .

Additional file 10: http://www.biomedcentral.com/imedia/ 1610501146675419/supp10.pdf. DATA SHEETS: O157-DMEM MS/MS data sheet 6 . 
Additional file 11: http://www.biomedcentral.com/imedia/ 1326109329675419/supp11.pdf. DATA SHEETS: O157-DMEM MS/MS data sheet 7 .

Additional file 12: http://www.biomedcentral.com/imedia/ 1285024576754199/supp12.pdf. DATA SHEETS: O157-DMEM MS/MS data sheet 8 .

\section{Competing interests}

The authors declare no competing financial interests.

\section{Acknowledgements}

This work was supported by a US National Institutes of Health Grant R2 Al055963 to I.T.K. Intellectual property rights for the 0157 proteome identified in this study are held by Massachusetts General Hospital, Boston, MA.

Excellent technical assistance provided by Bryan Wheeler at the National Animal Disease Center, Ames, IA, with the eukaryotic cell adherence/ adherence-inhibition assays is acknowledged.

\section{Disclaimer}

Mention of trade names or commercial products in this article is solely for the purpose of providing specific information and does not imply recommendation or endorsement by the U.S. Department of Agriculture. USDA is an equal opportunity provider and employer.

\section{Author details}

${ }^{1}$ Food Safety and Enteric Pathogens Research Unit, National Animal Disease Center, Agricultural Research Service, U.S. Department of Agriculture, Ames, lowa 50010, USA. ²Division of Infectious Diseases, Massachusetts General Hospital, Boston, Massachusetts 02114, USA. ${ }^{3}$ Harvard Partners Center for Genetics and Genomics, 65 Landsdowne Street, Cambridge, Massachusetts 02139, USA. ${ }^{4}$ Department of Medicine, Harvard Medical School, Boston, Massachusetts 02114, USA. ${ }^{5}$ Department of Microbiology and Molecular Genetics, Harvard Medical School, Boston, Massachusetts 02114, USA. ${ }^{6}$ Present Address: Thermo-Fisher Scientific, Cambridge, Massachusetts 02139 USA. ${ }^{7}$ Present Address: Pathovacs Inc., Ames, lowa 50010, USA.

\section{Authors' contributions}

ITK was the project leader and designed, coordinated, obtained funding, conducted experiments, analyzed data and drafted the manuscript. RWG conducted experiments and tabulated data. BK and DAS performed proteomic analysis. SBC assisted in design and participated in helpful discussions. MJ was the co-project leader, and designed, coordinated, analyzed results and performed bioinformatic analysis. All authors read and approved the final manuscript.

Received: 9 February 2012 Accepted: 12 June 2012

Published: 12 June 2012

\section{References}

1. Griffin PM, Ostroff SM, Tauxe RV, Greene KD, Wells JG, Lewis JH, Blake PA IIInesses associated with Escherichia coli 0157:H7 infections. A broad clinical spectrum. Ann Intern Med 1998, 109:705-712

2. Kaper JB, O'Brien AD: Escherichia coli O157:H7 and other shiga toxinproducing E. coli strains. Washington, D.C: ASM Press; 1998.

3. Scallan E, Hoekstra RM, Angulo FJ, Tauxe RV, Widdowson M, Roy SL, Jones \lrcorner , Griffin PM: Foodborne illness acquired in the United States -Major pathogens. Emerg Infect Dis 2011, 17:7-15

4. Vital signs: Incidence and trends of infection with pathogens transmitted commonly through food-Foodborne diseases active surveillance network, 10 U.S. Sites, 1996--2010. MMWR 2011, 60(22):749-755.

5. Kudva IT, Dean-Nystrom E: Bovine recto-anal junction squamous epithelial (RSE) cell adhesion assay for studying Escherichia coli 0157 adherence. J App Microbiol 2011, 111:1283-1294.

6. Li Y, Frey E, Mackenzie AMR, Finlay BB: Human response to Escherichia coli 0157:H7 infection: Antibodies to secreted virulence factors. Infect Immun 2000, 68:5090-5095.
7. Naylor SW, Low JC, Besser TE, Mahajan A, Gunn GJ, Pearce MC, MCKendrick IJ, Smith DG, Gally DL: Lymphoid follicle-dense mucosa at the terminal rectum is the principal site of colonization of enterohemmorhagic Escherichia coli 0157:H7 in the bovine host. Infect Immun 2003, 71:1505-1512

8. Naylor SW, Roe AJ, Nart P, Spears K, Smith DGE, Low JC, Gally DL: Escherichia coli 0157:H7 forms attaching and effacing lesions at the terminal rectum of cattle and colonization requires LEE4 operon. Microbiol 2005, 151:2773-2781.

9. Buchko SJ, Holley RA, Olson WO, Gannon VP, Veira DM: The effect of different grain diets on fecal shedding of Escherichia coli 0157:H7 by steers. J Food Prot 2000, 63:1467-1474.

10. Kudva IT, Hatfield PG, Hovde CJ: Effect of diet on the shedding of Escherichia coli 0157:H7 in a sheep model. Appl Environ Microbiol 1995 61:1363-1370.

11. Kudva IT, Jelacic S, Tarr PI, Youderian PA, Hovde CJ: Biocontrol of Escherichia coli 0157 with 0157-specific bacteriophages. Appl Environ Microbiol 1999, 65:3767-3773.

12. Murinda SE, Roberts RF, Wilson RA: Evaluation of colicins for inhibitory activity against diarrheagenic Escherichia coli strains, including serotype 0157:H7. Appl Environ Microbiol 1996, 62:3196-3202.

13. Nurmi E, Nuotio L, Schneitz C: The competitive exclusion concept: development and future. Int J Food Microbiol 1992, 15:237-240.

14. Zhao T, Doyle MP, Harmon BG, Brown CA, Mueller PO, Parks AH: Reduction of carriage of enterohemorrhagic Escherichia coli 0157:H7 in cattle by inoculation with probiotic bacteria. J Clin Microbiol 1998, 36:641-647.

15. Potter AA, Klashinsky S, Li Y, Frey E, Townsend H, Rogan D, Erickson G, Hinkley S, Klopfenstein T, Moxley RA, Smith DR, Finlay BB: Decreased shedding of Escherichia coli $0157: \mathrm{H} 7$ by cattle following vaccination with type III secreted proteins. Vaccine 2004, 22:362-369.

16. Allen KJ, Rogan D, Finlay BB, Potter AA, Asper DJ: Vaccination with type III secreted proteins leads to decreased shedding in calves after experimental infection with Escherichia coli 0157. Can J Vet Res 2011, 75:98-105.

17. Fox JT, Thomson DU, Drouillard JS, Thornton AB, Burkhardt DT, Emery DA, Nagaraja TG: Efficacy of Escherichia coli 0157:H7 siderophore receptor/ porin proteins-based vaccine in feedlot cattle naturally shedding $E$. coli 0157. Foodborne Path Dis 2009, 6:893-899.

18. Thomson DU, Loneragan GH, Thornton AB, Lechtenberg KF, Emery DA Burkhardt DT, Nagaraja TG: Use of a siderophore receptor and porin proteins-based vaccine to control the burden of Escherichia coli 0157:H7 in feedlot cattle. Foodborne Path Dis 2009, 6:871-877.

19. Carlson BA, Nightingale KK, Mason GL, Ruby JR, Choat WT, Loneragan GH, Smith GC, Sofos JN, Belk KE: Escherichia coli O157:H7 strains that persist in feedlot cattle are genetically related and demonstrate an enhanced ability to adhere to intestinal epithelial cells. App Environ Microbiol 2009, 75:5927-5937.

20. Sheng H, Wang J, Lim JY, Davitt C, Minnich SA, Hovde CJ: Internalization of Escherichia coli 0157:H7 by bovine rectal epithelial cells. Front Microbiol 2011, 2:1-32.

21. Perna NT, Plunkett G, Burland V, Mau B, Glasner JD, Rose DJ, Mayhew GF, Evans PS, Gregor J, Kirkpatrick HA, Posfai G, Hackett J, Klink S, Boutin A, Shao Y, Miller L, Grotbeck EJ, Davis NW, Lim A, Dimalanta ET, Potamousis KD, Apodaca J, Anantharaman TS, Lin J, Yen G, Schwartz DC, Welch RA, Blattner FR: Genome sequence of enterohaemorrhagic Escherichia coli 0157:H7. Nature 2001, 409:529-533.

22. McKee ML, O'Brien AD: Truncated enterohemmorhagic Escherichia coli (EHEC) 0157:H7 intimin (EaeA) fusion proteins promote adherence of EHEC strains to HEp-2 cells. Infect Immun 1996, 64:2225-2233.

23. Kudva IT, Krastins B, Sheng H, Griffin RW, Sarracino DA, Tarr PI, Hovde CI, Calderwood SB, John M: Proteomics-based expression library screening (PELS): a novel method for rapidly defining microbial immunoproteomes. Mol Cell Proteomics 2006, 5:514-519.

24. Peng J, Elias JE, Thoreen CC, Licklider LJ, Gygi SP: Evaluation of multidimensional chromatography coupled with tandem mass spectrometry (LC/LC-MS/MS) for large-scale protein analysis: the yeast proteome. J Proteome Res 2003, 2:43-50

25. Steen H, Mann M: The ABC's (and XYZ's) of peptide sequencing. Nat Rev Mol Cell Biol 2004, 5:699-711. 
26. John M, Kudva IT, Griffin RW, Dodson AW, McManus B, Krastins B, Sarracino D, Progulske-Fox A, Hillman JD, Handfield M, Tarr PI, Calderwood SB: Use of in vivo-induced antigen technology for identification of Escherichia coli 0157:H7 proteins expressed during human infection. Infect Immun 2005, 73:2665-2679

27. Sachdeva G, Kumar K, Jain P, Ramachandran S: SPAAN: a software program for prediction of adhesins and adhesin-like proteins using neural networks. Bioinformatics 2005, 15:483-491.

28. Mahajan A, Currie CG, Mackie S, Tree J, McAteer S, McKendrick I, McNeilly TN, Roe A, LaRagione RM, Woodward MJ, Glly DL, Smith DGE: An investigation of the expression and adhesin function of $\mathrm{H} 7$ flagella in the interaction of Escherichia coli 0157:H7 with bovine intestinal epithelium. Cell Microbio/ 2009, 11:121-137.

29. Xicohtencatl-Cortes J, Chacon ES, Saldana Z, Freer E, Giron JA: Interaction of Escherichia coli 0157:H7 with leafy green produce. J Food Protect 2009, 72:1531-1537.

30. Fagerquist CK, Garbus BR, Miller WG, Williams KE, Yee E, Bates AH, Boyle S, Harden LA, Cooley MB, Mandrell RE: Rapid identification of protein biomarkers of Escherichia coli 0157:H7 by matrix-assisted laser desorption ionization-time-of-flight - time-of-flight mass spectrometry and top-down proteomics. Anal Chem 2010, 82:2717-2725.

31. Gunther NW, Pang H, Nunez A, Uhlich GA: Comparative proteomics of E. coli 0157:H7: Two-dimensional gel electrophoresis vs. twodimensional liquid chromatography separation. The Open Proteom J 2010, 3:26-34

32. Tremoulet F, Duche O, Namane A, Martinie B, Labadie JA: Proteomic study of Escherichia coli 0157:H7 NCTC 12900 cultivated in biofilm or in planktonic growth mode. FEMS Microbiol Lett 2002, 215:7-14.

33. Zheng S, Schneider KA, Barder TJ, Lubman DM: Two-dimensional liquid chromatography protein expression mapping for differential proteomic analysis of normal and 0157:H7 Escherichia coli. Biotechniq 2003, 35:1202-1212.

34. Sperandio V, Torres AG, Jarvis B, Nataro JP, Kaper JB: Bacteria-host communication: The language of hormones. PNAS 2003, 100:8951-8956.

doi:10.1186/1471-2180-12-103

Cite this article as: Kudva et al: Proteins other than the locus of enterocyte effacement-encoded proteins contribute to Escherichia coli O157:H7 adherence to bovine rectoanal junction stratified squamous epithelial cells. BMC Microbiology 2012 12:103.

\section{Submit your next manuscript to BioMed Central and take full advantage of:}

- Convenient online submission

- Thorough peer review

- No space constraints or color figure charges

- Immediate publication on acceptance

- Inclusion in PubMed, CAS, Scopus and Google Scholar

- Research which is freely available for redistribution 